\title{
ON THE CORRECTION OF THE POWER FACTOR IN MODERN LOW-VOLTAGE POWER ELECTRICAL INSTALLATIONS
}

\author{
EMIL CAZACU ${ }^{1}$, MARILENA STĂNCULESCU $^{1}$, HORIA ANDREI $^{2}$ \\ ${ }^{1}$ University POLITEHNICA of Bucharest, Romania, Faculty of Electrical Engineering, \\ 313, Splaiul Independentei, 060042, district 6, Bucharest ROMANIA \\ ${ }^{2}$ University VALAHIA of Bucharest, ROMANIA, Doctoral School of Engineering Sciences \\ E-mail: emil.cazacu@upb.ro, marilena.stanculescu@upb.ro, horia.andrei@valahia.ro
}

\begin{abstract}
The paper presents the main electrical loads that require reactive power, evaluating also its value in different operating states of the industrial installation (permanent sinusoidal or distorted). Also, the effects of reactive power flow in the networks are quantitatively estimated and accordingly, a series of specific power quality indicators (e.g. power factor, crest factor, total harmonic distortion) are highlighted. Different types of solutions dedicated to reduce the level of reactive power are also suggested. The proper design of the power factor correction systems is directly correlated with the consumer power quality parameters. A case-study will round up the work, underling the major importance of the reactive power flow in modern electric installations.
\end{abstract}

Keywords: reactive power, power factor, power quality, harmonics

\section{INTRODUCTION}

In order to fulfill their functional role, AC electrical receivers absorb from the network generally, in addition to active power (the useful power) also reactive power required by the receiver according to their character (inductive or more rarely, capacitive) [1-5]. Supplementary, nonlinear consumers (receivers with an increasing incidence in modern network) require, besides active and reactive powers, also the distorting power - as a result of nonsinusoidal waveform of current and/or voltage [4-8]. The circulation of reactive and distorted power causes additional losses in equipment and network, affecting significantly power quality and energy efficiency of installations and the entire process of energy transfer from the source to the loads (consumers). It is therefore required a complete control and management of the reactive and distorted power flow in the installation at all its levels [7-14].

The paper presents the major electrical loads that demand reactive power and operate in permanent steady-state (either sinusoidal or nonsinusoidal) regime. The main adverse effects of reactive power flow in the network are highlighted, suggesting also effective mitigation solution according to the level of different power quality indicators (mainly harmonic distortion factors). Thus, the power factor correction equipment (various types of capacitor banks, passive or active filters, etc.) is designed according to the load power quality indicators, that are continuously measured and analyzed. Finally, the favorable consequences for a load operating with a higher power factor are highlighted for a typical high power industrial load.

\section{THE MAIN REACTIVE POWER LOADS IN ELECTRIC INSTALLATIONS}

The main reactive power consumers are the asynchronous motors and the electrical transformers, which consume around $60 \%$, respectively $25 \%$ from the installation's total reactive power [9-14]. It is estimated that at industrial consumer's level, the reactive power receivers is around $70 \%$ for asynchronous motors and around $20 \%$ for transformers [13]. Another component of the reactive power for the asynchronous motors and for transformers is the reactive power dependent on load, called also dispersion reactive power.

Taking into account the exploitation average loading $\beta<0.5$ (evaluated using the loading factor as the ratio between the mechanical shaft power $P$ and mechanical nominal power $P_{n}$ of the motor $\left.\beta=P / P_{n}\right)$, one can approximate the reactive power of an asynchronous motor as being constant and independent of the load, while the active power depends on the motor's load. Also, the motors' active power remains practically constant for small deviations of the voltage compared to the nominal voltage, while the reactive power depends essentially on the voltage's variation. Asynchronous motors functioning with a load factor $\beta<1$ due to an inappropriate technological exploitation, determines the power factor reduction under its nominal value. The reactive power $Q$ absorbed by an asynchronous motor for any load $P$, is determined using the relationship [12-15]:

$$
Q=Q_{n}\left[\alpha+(1-\alpha) \beta^{2}\right]=Q_{0}+\left(Q_{n}-Q_{0}\right) \beta^{2},
$$

where $\alpha=Q_{0} / Q_{n}$ the ratio is between the reactive power $\mathrm{Q}_{0}$ for unloaded running $(\beta=0)$ and the reactive power $Q_{n}$ absorbed at nominal load $(\beta=1)$; $Q_{d}=(1-\alpha) \beta^{2} Q_{n} \quad-$ dispersion reactive power. Considering the definition of the power factor (PF) in sinusoidal state, for a symmetrical loading of the 3-phase network, it results [8-14]: 


$$
\begin{aligned}
& \lambda=P F=\cos \varphi= \\
& =\frac{\beta}{\sqrt{\beta^{2}+\left[\alpha+(1-\alpha) \beta^{2}\right]^{2} \tan ^{2} \varphi_{n}}}
\end{aligned}
$$

For values of $\beta=0.5$, the reduction of the PF under its nominal value is highly accentuated. If in exploitation, the power voltage of the asynchronous motors increases, it results an increase of the absorbed reactive power, having undesired consequences upon PF - Figure 1-a. This is due to the increase of the magnetization current in the saturated area. In Figure 1-b is presented, as a rough guide, the dependence of the absorbed reactive power function of the nominal one ( $Q / Q_{n}$ ) for asynchronous motors of low and high power function of the relative value of the power voltage for different loading factor's values [12-15].

For transformers the absorbed reactive power is computed using the relationship:

$$
Q=Q_{0}+Q_{d}=\frac{S_{n}}{100}\left(i_{0}[\%]+k_{f} \beta^{2} u_{s c}[\%]\right)
$$

where $S_{n}$ is the nominal apparent power, $i_{0}[\%]-$ the current for unloaded running (expressed in percentage function of nominal current), $k_{f}$ - the form factor of the load profile (defined as the ratio between the mean square value and the mean value, of the load current, computed for a certain time interval), $\beta=S / S_{n}-$ the transformer load factor, $u_{s c}[\%]-$ the voltage short-circuit voltage (expressed in percents).
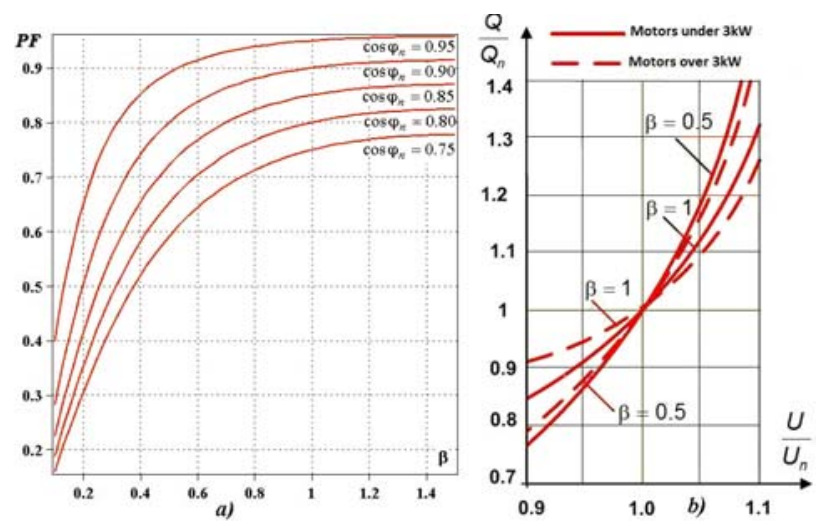

Figure 1. Reactive power dependency of different parameters.

As for asynchronous motors case, the transformers functioning at a power under the nominal one determines the reduction of PF. In real exploitation conditions the transformer's total reactive power can be evaluated to $10 \%$ of the nominal power ( $8 \%$ unloaded running power and $2 \%$ dispersion power).

The modern consumers are nonlinear; accordingly their voltage and current waveform are nonsinusoidal and can be easily expressed according to Fourier series [5-17]:

$$
\begin{aligned}
& u(t)=\sum_{k=1}^{n} \sqrt{2} U^{(k)} \sin \left(k \omega t+\varphi_{U k}\right) ; \\
& i(t)=\sum_{k=1}^{n} \sqrt{2} I^{(k)} \sin \left(k \omega t+\varphi_{I k}\right)
\end{aligned}
$$

where $k$ is the harmonic order.

The active $\mathrm{P}$, reactive $\mathrm{Q}$ and distorted $\mathrm{D}$ power of a comprising harmonic load is the sum of the active respectively reactive powers resulting from the currents and voltages of the same order [1-5]:

$$
\begin{gathered}
P=\sum_{k=1}^{n} U^{(k)} I^{(k)} \cos \varphi_{k} \\
Q=\sum_{k=1}^{n} U^{(k)} I^{(k)} \sin \varphi_{k} \\
D=\sqrt{S^{2}-P^{2}-Q^{2}}
\end{gathered}
$$

where $\mathrm{S}$ is the apparent power expressed as a function of RMS (root mean square) voltage and current: $S=U_{\mathrm{RMS}} I_{\mathrm{RMS}}$.

The power factor PF under these circumstances is defined as the ratio between the active power $\mathrm{P}$ and the apparent power S, while the displacement power factor (DPF) also known as (defined exclusively in terms of the fundamental $\mathrm{k}=1) \cos \varphi_{1}$, are:

$$
P F=\frac{P}{S}, \quad D P F=\cos \varphi_{1}=\frac{P_{1}}{S_{1}}
$$

For installation with high value of the short-circuit power at common coupling point, the voltage distortion is insignificant and the ratio of power factor and displacement power factor can be expressed in terms of current total harmonic distortion $\left(\mathrm{THD}_{\mathrm{I}}\right)$. The latter is widely used for expressing the level of harmonic content of a distorted waveform [12-17]:

$$
\begin{gathered}
P F=\frac{\cos \varphi_{1}}{\sqrt{1+T H D_{I}^{2}}} \\
\text { with } T H D_{I}=\sqrt{\sum_{k=2}^{\infty} I_{k}^{2}} / I_{1} .
\end{gathered}
$$

The variation ratio of $\frac{P F}{\cos \varphi_{1}}$ in respect to percentage of $T H D_{I}$, when $T H D_{U}$ is unconsidered is quantitatively represented in Figure 2. 


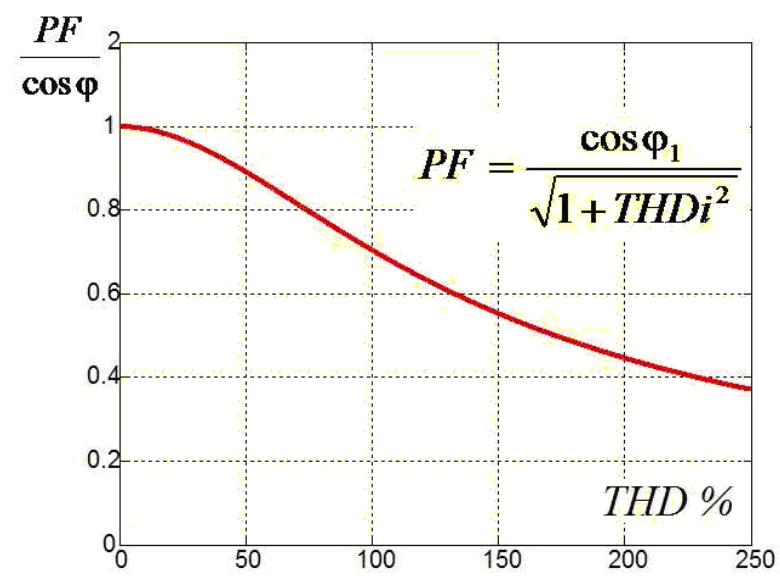

Figure 2. The ratio $P F / \cos \varphi_{1}$ variation in respect to $T H D_{I}$ in the case when $T H D_{U}=\mathbf{0}$.

\section{REACTIVE POWER FLOW ADVERSE CONSEQUENCES}

The irrational reactive power consumption generates a reduced power factor that leads to numerous undesirable consequences for the entire electrical installation [10-14]:

a) The increase of active power losses $\Delta P$ in the passive elements of resistance $R$ in the installation:

$$
\Delta P=3 R I^{2}=\frac{R}{U^{2}} S^{2}=\frac{R P^{2}}{U^{2} P F^{2}}
$$

considering two different power factors $P F_{1}$ and $P F_{2}$ the corresponding power absorbed currents $I_{1}, I_{2}$ and conductor's losses $\triangle P_{1}$ and $\Delta P_{2}$ are connected by the relation: $\quad I_{2}=I_{1}\left(P F_{1} / P F_{2}\right) \Delta P_{2}=\Delta P_{1}\left(P F_{1} / P F_{2}\right)^{2}$. Accordingly, one can notice that by improving the power factor, the power losses significantly diminishes.

b) Effective value voltage alternations. In the sinusoidal state, for an inductive power factor, the longitudinal voltage drop $\Delta U=U_{1}-U_{2}$ is given by the relationship:

$$
\begin{aligned}
& \Delta U=R I \cos \varphi+X I \sin \varphi= \\
& =\frac{R \cdot P+X \cdot Q}{U_{2}}=\Delta U_{a}+\Delta U_{r}
\end{aligned}
$$

where $U_{1}$ is the phase voltage at the power source terminals, $U_{2}$ is the phase voltage at the consumers, $R$ and $X$ represents the electric resistance and he reactance of the line that connects the source to the receiver respectively.

As a consequence, when $Q=0$, one obtains the minima voltage drop: $\Delta U=\Delta U_{\min }=\Delta U_{a}$.

c) Diminishing the installation's active power loading capacity due to a reduced power factor.
A facility with a certain apparent power $\mathrm{S}_{\mathrm{n}}$ may deliver, a certain active powers: $P_{1}=S_{n} P F_{1}, P_{2}=S_{n} P F_{2}$ according to the power factor value. If $P F_{2}<P F_{1}$ then we have: $P_{2}=\left(P F_{2} / P F_{1}\right) P_{1}$, from where it results active power reduction $P_{2}<P_{1}$, increasing the reactive power consumption.

d) Electric installation's oversize. Taking into account the admissible voltage losses $\Delta U_{r}$ expressed in terms of its active $\Delta U_{a}$ and reactive part $\Delta U_{r}$ (under sinusoidal state):

$$
\Delta U_{a d}=\Delta U_{a}+\Delta U_{r}=\rho \frac{L}{S} \frac{P}{U_{n}}+\frac{X \cdot Q}{U_{n}}
$$

with: $\mathrm{L}$ the length of the line; $\mathrm{s}$ the phase conductor's section; $\rho$ the resistivity of the conducting material; P , Q - the active and reactive power flow, respectively.

One can derive:

$$
\begin{aligned}
& \Delta U_{a}=\Delta U_{a d}-\Delta U_{r}=\rho \frac{L}{s} \frac{P}{U_{n}}=c t . \\
& \text { or } s=\rho \frac{L}{\Delta U_{a}} \frac{P}{U_{n}}
\end{aligned}
$$

Thus, the section of the conductive elements is directly connected with the reactive power flow into the installation: greater the amounts of reactive power, thicker conductors are required.

\section{REACTIVE POWER MITIGATION SOLUTION}

The most common procedures to improve the power factor $(\mathrm{PF})$ are grouped into natural procedures and special procedures. The natural methods derive from a rational and correct choice and exploitation of the existing machinery in installations and mainly consist in technical and managerial means (replacing the low-loaded transformers and motors, nonlinear loads aggregation, reducing the unloaded operating time of the machineries etc.). The special ones assume the introduction in installation of some equipments generating reactive power and/or limiting the distorted state (installation of some capacitors banks, of active filters networks conditioners etc.). As a consequence, PF improvement includes, on a first stage, operations for mitigation the high harmonics' attendance from the wave shapes of the voltage and the absorbed current, and, on the second stage, the limitation of reactive power flow. Using the capacitor bank to reduce the reactive power absorbed by a consumer is efficient only if the power system voltage is practically sinusoidal.

To select a system for power factor correction in an installation where there are present high-order harmonics in the current and voltage waves shape, function of the user's data, one uses the following methods [1-16]:

a. Distorted installed power procedure, whose principle is described in Figure 3. The following notations were used: 
- $G$ is the sum of apparent powers taken for all equipments that generate harmonics (power static converters, inverters variable speed drives, etc.) connected to the mains where the capacitors bank is also connected. If, for some equipment the active power is given, to compute the apparent power one should take into consideration the covering $P F$.

- $S_{n}$ is the sum of apparent powers taken for all system's power transformers to which the distribution mains belong.

The above method can be applied only when one has a limited voltage and current harmonic distorted level $\left(T H D_{U}<5 \%, T H D_{I}<30 \%\right)$.
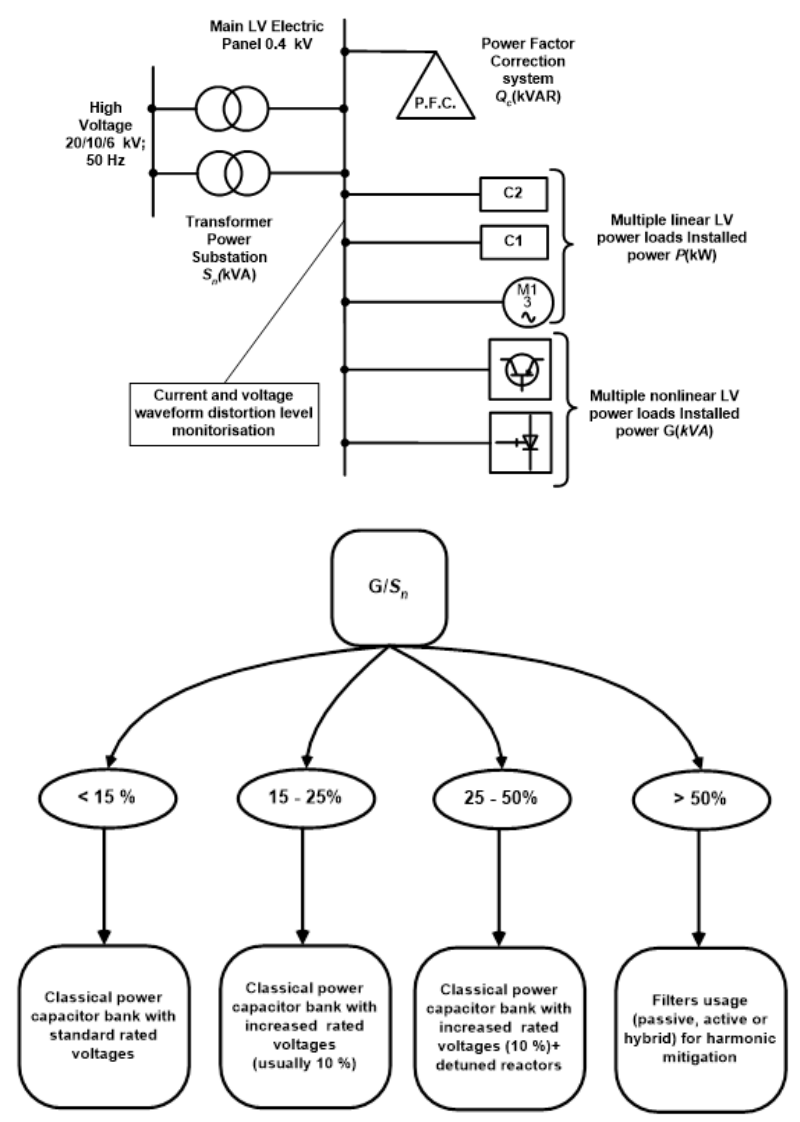

Figure 3. Selecting the reactive power compensation method according to the installation's nonlinear receivers' level relative to the installation apparent power.

b. Voltage and Current Total Harmonic Distorted Level Determination Method, applicable when one knows exactly (by measurements) the values corresponding to $T H D_{U}$ respectively to $T H D_{I}$. The principle of this method is presented in Figure 4.

The selection methods indicate the usage of (depending on the harmonic's distortion level): standards capacitor banks, capacitor banks with increased nominal voltage (usual with $10 \%$ ), capacitor banks with increased nominal voltage and with detuned reactors or harmonic mitigation filters (active, passive or hybrid).

One should mention that in actual conditions, where the installation's load with a voltage or especially current highorder harmonics are very frequent, capacitor banks placed in series with certain coils also called "detuned reactors" shown in Figure 5 [5-17], are used as a power factor correction solution (cheaper than using active filters).

The reactive power corresponding to these detuned reactors is chosen usually as a percent, called detuned reactor factor, usually $\mathrm{p}=5 \%, 7 \%$ or $11 \%$ from the compensation reactive power of the bank. Therefore, by a correct selection of the detuned reactor factor, one may avoid the resonance on the dominant installation's harmonics and at the fundamental frequency the compensation function is satisfactory maintained.

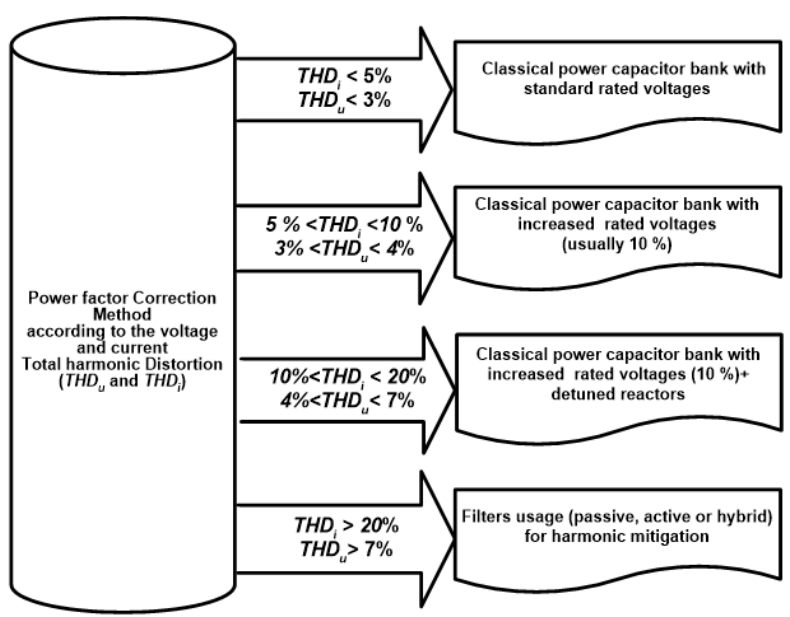

Figure 4. Selection of reactive power compensation method according to the voltage and current total harmonic distortion (THD) level.

Moreover, the detuned reactors, that have values of $\mathrm{mH}$ order, together with the connecting conductors' inductivity, limit the commutation inrush currents in the case of switching the capacitor banks from automatic power factor correction system (APFC). Moreover, the detuned reactors supplementary stress the capacitor's dielectric. Consequently, they should be dimensioned at voltages at least $10 \%$ bigger than the nominal ones.

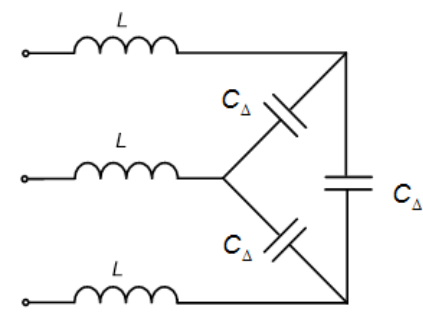

Figure 5. Connecting the detuned reactors in a $\Delta$ connection to a capacitors bank.

The reduction of the inrush current value is simply done by placing in series with the capacitors very small inductivity coils (a few $\mu \mathrm{H}$ ). For low-voltage banks where the harmonics do not have a high consistent ( $T H D_{U, I}<10 \%$ ) even the power cable twisted in several turns realizes a current limitation. So, only the inductivity of a $10 \mathrm{~m}$ cable is sufficient to limit the inrush currents cause by connecting a capacitor bank of a power up to $25 \mathrm{kVAr}$ [8-14]. 
These measures should determine the consumers to operate with an exploitation power factor at values very close to the nominal one and finally to lead to an increased average medium power factor at least up to the neutral value (usually $0.92 \div 0.98$ ).

\section{CASE STUDY}

We will consider an industrial facility with numerous electric linear power consumers (most of them induction motors with a variety of power rates). The main power quality parameters, measured at the receivers' common coupling point (low-voltage part of a $400 \mathrm{kVA}-20 / 0.4 \mathrm{kV}$ distribution transformer) are presented in Figure 6.

The captures are recorded by a Fluke 435 a class A three phase power quality analyzer [18]. Since the voltage and current harmonics as well as the unbalance level are insignificant, a power factor correction with simple capacitors banks is to be designed. One can notice that the actual (measured) power factor $P F_{1}=\cos \varphi_{1}=0.76$ is unsatisfactory and a new higher one is required. Consequently, a capacitor bank system is evaluated in order to maintain a value of $P F_{2}=\cos \varphi_{2}=0.98$ in the transformer secondary winding. The active power $P=334.4 \mathrm{~kW}$ imposes the capacitor banks Reactive power:

$$
\begin{aligned}
& Q_{b a t}=P \cdot\left(\operatorname{tg} \varphi_{1}-\operatorname{tg} \varphi_{2}\right)=166.25 \cdot(0.85-0.203) \cong \\
& \cong 218.26 \mathrm{kVAr}
\end{aligned} .
$$

An automatic power factor correction system (fractional multiple steps capacitor banks) of power value: $Q_{\text {Bank }}=220 \mathrm{kVAr}$. A solution of the automatic capacitor bank can be realized in six steps of 50, 50, 30, 30, 30 and $30 \mathrm{kVAr}$ respectively. The new current absorbed by the consumer $I_{2}$ and the power losses $\Delta P_{2}$ in the supply up to the receiver could be expressed in terms of their initial value $I_{1}$ and $\Delta P_{1}$ respectively:

$$
\begin{gathered}
I_{2}=I_{1} \frac{P F_{1}}{P F_{2}}=0.77 I_{1}, \\
\Delta P_{2}=\Delta P_{1}\left(\frac{P F_{1}}{P F_{2}}\right)^{2}=0.59 \Delta P_{1}
\end{gathered}
$$
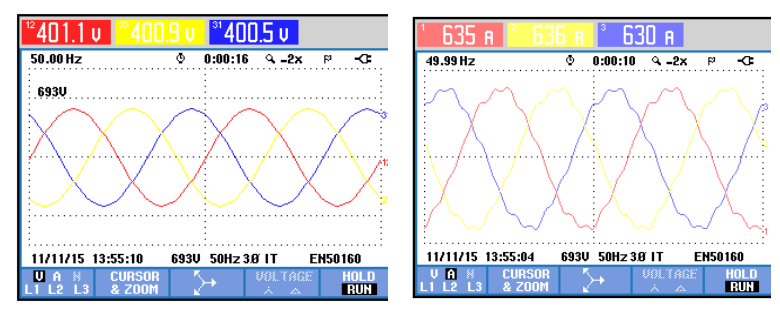
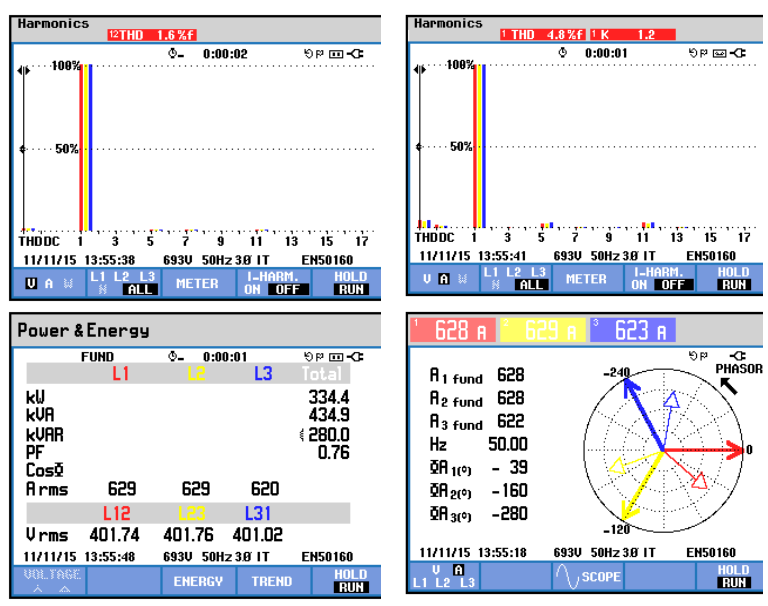

Figure 6. The main power quality parameters of the investigated industrial consumer.

One can notice the importance of the power factor correction can could lead to a considerable reduction of the absorbed current (almost $25 \%$ ) and the power losses on the distribution line (over $35 \%$ ).

\section{CONCLUSIONS}

The reactive power control has a significant role in supporting the power transmission systems. Reactive power is required to deliver active power through transmission lines at a certain voltage level. Motors, transformers and other power loads, intrinsically require reactive power in order to maintain their functionality. Either the lake or the excess of reactive power may lead to numerous power quality disturbances, which adversely affect both the loads and the supply network.

The power factor correction equipment is to be selected only after a rigorous analysis of power quality in the installations where the reactive power is to be compensated. This implies, in principle, to master all the power quality parameters, especially those which express the level of harmonic voltage and current distortion. That could be nowadays easily performed by the modern portable power quality analyzers. More over, the costs of these measurements devices is decreasing, making them suitable for large range of electric installations.

Additionally, the installation owner should also take into account the economic aspects of such corrective means and constantly analyze the payback of this investment. Under current conditions, a recovery period of one year is considered acceptable.

The principal methods used especially in existing installations are:

- Power transformers running in parallel, following the minimum reactive losses graph, when the exploitation conditions allow it;

- Exploitation of synchronous motors at the maximum limit of producing reactive power; 
- Limiting the unloaded running of asynchronous motors, of special transformers, if the technological process allows it;

- Using Y- $\Delta$ switches for low-voltage asynchronous motors, systematically loaded under $40 \%$ from its nominal load, for a long-term functioning in $\mathrm{Y}$ connection;

- $\quad$ Replacing asynchronous motors and of the over-sized transformers, based on technical-economical analysis using the updated total spending method.

The most efficient, but still expensive, power factor correction method in modern electric installations is the usage of active filters, known also as Active Harmonic Conditioners (AHC). These are basically static power converters that can also perform additional functions in the network (such as reducing the harmonic content and electrically balance the receiver).

\section{REFERENCES}

[1] Saeed Anwar, Ali Elrayyah, Yilmaz Sozer, Efficient single phase power factor improvement strategy for microgrid operation, Applied Power Electronics Conference and Exposition (APEC) 2014 TwentyNinth Annual IEEE, pp. 972-977, 2014.

[2] J. Dixon, L. Moran, J. Rodriguez, R. Domke, Reactive power compensation technologies: stateof-the-art review, Proceedings of the IEEE, vol. 93, no. 12, pp. 2144-2164, Dec. 2005.

[3] A. Pana, A. Baloi, F. Molnar-Matei, Load balancing by unbalanced capacitive shunt compensation - A numerical approach, Harmonics and Quality of Power (ICHQP) 2010 14th International Conference on, pp. 1-6, 2010.

[4] S. Krishnamurthy, G.F. Noudjiep Djiepkop, Performance analysis and improvement of a power system network using a Unified Power Flow Controller, Industrial and Commercial Use of Energy (ICUE) 2015 International Conference on the, pp. 306-312, 2015.
[5] Uday B. Mujumdar, Jayant S. Joshi, Optimal reactive power compensation under non sinusoidal conditions using current minimization method, Power and Energy Systems (ICPS) 2011 International Conference on, pp. 1-6, 2011.

[6] Rashid H. M., Power Electronics: Circuits, Devices \& Applications, Prentice Hall; 4th Edition, 2013.

[7] Bisanovic S. , Hajro M., Samardzic M., One approach for reactive power control of capacitor banks in distribution and industrial networks, International Journal of Electrical Power \& Energy Systems, vol. 60, pp. 67, 2014.

[8] Hofmann W., Schlabbach J., Just W., Reactive Power Compensation: A Practical Guide, John Wiley \& Sons, 2012.

[9] Schneider Electric, Electrical Installation Guide, 2016.

[10] Ferracci, Ph. Power Quality, Schneider Electric, Cahier Technique no. 199, 2001.

[11] ABB, Power factor correction and harmonic filtering in electrical plants, Technical Application Paper, 2008.

[12] Dugan, R. C., McGranaghan, M. F., Santoso, S., Beaty H. W., Electrical Power Systems Quality, McGraw Hill Professional, 2012.

[13] Cazacu E., Instalaţii electrice moderne (in Romanian), Editura Matrix-Ronm, 2016.

[14] Cazacu E., Petrescu L., Expertiza sistemelor electrice industriale (in Romanian), Editura Printech, 2014.

[15] D. J. Carnovale, T. J. Dionise, and T. M. Blooming, Price and performance considerations for harmonic solutions, in Proc. Power Syst. World, Power Quality Conf., Long Beach, CA, 2003.

[16] Emanuel A. E., Power Definitions and the Physical Mechanism of Power Flow, John Wiley \& Sons, IEEE Press, 2011.

[17] Bettega, E., Fiorina J-N., Active harmonic conditioners and unity power factor rectifiers, Schneider Electric, Cahier Technique no. 183, 1999.

[18] *** Fluke 435 -class A - Three-phase power quality analyzer - Operating manual, Fluke Corporation, 2009. 